\title{
Twisted Alexander polynomials detect the unknot
}

\author{
DANIEL S SILVER \\ SUSAN G WiLliams
}

\begin{abstract}
The group of a nontrivial knot admits a finite permutation representation such that the corresponding twisted Alexander polynomial is not a unit.
\end{abstract}

$57 \mathrm{M} 25 ; 37 \mathrm{~B} 40$

\section{Introduction}

Twisted Alexander polynomials of knots in $\mathbb{S}^{3}$ were introduced by X-S Lin in [7]. They were defined more generally for any finitely presentable group with infinite abelianization by M Wada [11]. Many papers subsequently appeared on the topic. Notable among them is [5], by P Kirk and C Livingston, placing twisted Alexander polynomials of knots in the classical context of abelian invariants. A slightly more general approach by $\mathrm{J}$ Cha [1] permits coefficients in a Noetherian unique factorization domain.

In Hillman-Livingston-Naik [4] two examples are given of Alexander polynomial 1 hyperbolic knots for which twisted Alexander polynomials provide periodicity obstructions. In each case, a finite representation of the knot group is used to obtain a nontrivial twisted polynomial. Such examples motivate the question: Does the group of any nontrivial knot admit a finite representation such that the resulting twisted Alexander polynomial is not a unit (that is, not equal to $\pm t^{i}$ )?

Theorem Let $k \subset \mathbb{S}^{3}$ be a nontrivial knot. There exists a finite permutation representation such that the corresponding twisted Alexander polynomial $\Delta_{\rho}(t)$ is not a unit.

A key ingredient of the proof of the theorem is a recent theorem of M Lackenby [6] which implies that some cyclic cover of $\mathbb{S}^{3}$ branched over $k$ has a fundamental group with arbitrarily large finite quotients. The quotient map pulls back to a representation of the knot group. A result of J Milnor [8] allows us to conclude that for sufficiently large quotients, the associated twisted Alexander polynomial is nontrivial. 
We are grateful to Abhijit Champanerkar and Walter Neumann for helpful discussions. We thank the referee for many thoughtful suggestions that improved the exposition of the paper. Both authors were partially supported by NSF grant DMS-0304971.

\section{Preliminary material}

\subsection{Review of twisted Alexander polynomials}

Let $X$ be a finite CW complex. Its fundamental group $\pi=\pi_{1} X$ acts on the left of the universal cover $\tilde{X}$ by covering transformations.

Assume that $\epsilon$ is an epimorphism from $\pi$ to an infinite cyclic group $\langle t \mid\rangle$. Given a Noetherian unique factorization domain $R$, we identify the group ring $R[\langle t \mid\rangle]$ with the ring of Laurent polynomials $\Lambda=R\left[t, t^{-1}\right]$. (Here we will be concerned only with the case $R=\mathbb{Z}$.)

Assume further that $\pi$ acts on the right of a free $R$-module $V$ of finite rank via a representation $\rho: \pi \rightarrow G L(V)$. Define a $\Lambda-R[\pi]$ bimodule structure on $\Lambda \otimes_{R} V$ by $t^{j}\left(t^{n} \otimes v\right)=t^{n+j} \otimes v$ and $\left(t^{n} \otimes v\right) g=t^{n+\epsilon(g)} \otimes v \rho(g)$ for $v \in V$ and $g \in \pi$. The groups of the cellular chain complex $C_{*}(\tilde{X} ; R)$ are left $R[\pi]$-modules. The twisted complex of $X$ is defined to be the chain complex of left $\Lambda$-modules:

$$
C_{*}(X ; \Lambda \otimes V)=(\Lambda \otimes V) \otimes_{R[\pi]} C_{*}(\tilde{X} ; R) .
$$

The twisted homology $H_{*}(X ; \Lambda \otimes V)$ is the homology of $C_{*}(X ; \Lambda \otimes V)$.

Since $V$ is finitely generated and $R$ is Noetherian, $H_{*}(X ; \Lambda \otimes V)$ is a finitely presentable $\Lambda$-module. Elementary ideals and characteristic polynomials are defined in the usual way. Begin with an $n \times m$ presentation matrix corresponding to a presentation for $H_{1}(X ; \Lambda \otimes V)$ with $n$ generators and $m \geq n$ relators. The ideal in $\Lambda$ generated by the $n \times n$ minors is an invariant of $H_{1}(X ; \Lambda \otimes V)$. The greatest common divisor of the minors, the twisted Alexander polynomial of $X$, is an invariant as well. It is well defined up to a unit of $\Lambda$. Additional details can be found in [1]. An alternative, group-theoretical approach can be found in [9].

In what follows, $X$ will denote the exterior of a nontrivial knot $k$, that is, the closure of $\mathbb{S}^{3}$ minus a regular neighborhood of $k$.

\subsection{Periodic representations}

The knot group $\pi$ is a semidirect product $\langle x \mid\rangle \ltimes \pi^{\prime}$, where $x$ is a meridional generator and $\pi^{\prime}$ denotes the commutator subgroup $[\pi, \pi]$. Every element has a unique expression of the form $x^{j} w$, where $j \in \mathbb{Z}$ and $w \in \pi^{\prime}$. 
For any positive integer $r$, the fundamental group of the $r$-fold cyclic cover $X_{r}$ of $X$ is isomorphic to $\left\langle x^{r} \mid\right\rangle \ltimes \pi^{\prime}$. The fundamental group of the $r$-fold cyclic cover $M_{r}$ of $\mathbb{S}^{3}$ branched over $k$ is the quotient group $\pi_{1}\left(X_{r}\right) /\left\langle\left\langle x^{r}\right\rangle\right\rangle$, where $\langle\langle\cdot\rangle\rangle$ denotes the normal closure. Consequently, $\pi_{1} M_{r}=\pi^{\prime} /\left[\pi^{\prime}, x^{r}\right]$.

Definition 2.1 A representation $p: \pi^{\prime} \rightarrow \Sigma$ is periodic with period $r$ if it factors through $\pi_{1} M_{r}$. If $r_{0}$ is the smallest such positive number, then $p$ has least least period $r_{0}$.

Remark 2.2 The condition that $p$ factors through $\pi_{1} M_{r}$ is equivalent to the condition that $p\left(x^{-r} a x^{r}\right)=p(a)$ for every $a \in \pi^{\prime}$.

The following is a consequence of the fact that $M_{1}$ is $\mathbb{S}^{3}$.

Proposition 2.3 If $p: \pi^{\prime} \rightarrow \Sigma$ has period 1, then $p$ is trivial.

Assume that $p: \pi^{\prime} \rightarrow \Sigma$ is surjective and has least period $r_{0}$. We extend $p$ to a homomorphism $P: \pi \rightarrow\left\langle\xi \mid \xi^{r_{0}}\right\rangle \ltimes_{\theta} \Sigma^{r_{0}}$, mapping $x \mapsto \xi$ and elements $u \in \pi^{\prime}$ to $\left(p(u), p\left(x^{-1} u x\right), \ldots, p\left(x^{-\left(r_{0}-1\right)} u x^{r_{0}-1}\right)\right) \in \Sigma^{r_{0}}$. Conjugation by $\xi$ in the semidirect product induces $\theta$ : $\Sigma^{r_{0}} \rightarrow \Sigma^{r_{0}}$ described by $\left(\alpha_{1}, \ldots, \alpha_{r_{0}}\right) \mapsto\left(\alpha_{2}, \ldots, \alpha_{r_{0}}, \alpha_{1}\right)$. The lemma below assures us that the image of $\pi^{\prime}$ under $P$ has order no less than the order of $p\left(\pi^{\prime}\right)$.

Lemma $2.4\left|P\left(\pi^{\prime}\right)\right| \geq\left|p\left(\pi^{\prime}\right)\right|$

Proof The image $P\left(\pi^{\prime}\right)$ is contained in $\Sigma^{r_{0}}$. First coordinate projection $\Sigma^{r_{0}} \rightarrow \Sigma$ obviously maps $P\left(\pi^{\prime}\right)$ onto $p\left(\pi^{\prime}\right)$.

In what follows we will assume that $\Sigma$ is finite. Hence $P(\pi)$ is also finite, and it is isomorphic to a group of permutations of a finite set acting transitively (that is, the the orbit of any element under $P(\pi)$ is the entire set.) We can ensure that the subgroup $P\left(\pi^{\prime}\right)$ also acts transitively, as the next lemma shows.

We denote the symmetric group on a set $\mathcal{A}$ by $S_{\mathcal{A}}$.

Lemma 2.5 The group $P(\pi)$ embeds in the symmetric group $S_{P\left(\pi^{\prime}\right)}$ in such a way that $P\left(\pi^{\prime}\right)$ acts transitively. 
Proof Embed $P\left(\pi^{\prime}\right)$ in $S_{P\left(\pi^{\prime}\right)}$ via the right regular representation $\psi: P\left(\pi^{\prime}\right) \rightarrow$ $S_{P\left(\pi^{\prime}\right)}$. Given $\beta=\left(\beta_{1}, \ldots, \beta_{r_{0}}\right) \in P\left(\pi^{\prime}\right)$, the permutation $\psi(\beta)$ maps $\left(\alpha_{1}, \ldots, \alpha_{r_{0}}\right) \in$ $P\left(\pi^{\prime}\right)$ to $\left(\alpha_{1} \beta_{1}, \ldots, \alpha_{r_{0}} \beta_{r_{0}}\right)$. Extend $\psi$ to $\Psi: P(\pi) \rightarrow S_{P\left(\pi^{\prime}\right)}$ by assigning to $\xi$ the permutation of $P\left(\pi^{\prime}\right)$ given by $\left(\alpha_{1}, \ldots, \alpha_{r_{0}}\right) \Psi(\xi)=\left(\alpha_{2}, \ldots, \alpha_{r_{0}}, \alpha_{1}\right)$. It is straightforward to check that $\Psi$ respects the action $\theta$ of the semidirect product, and hence is a well-defined homomorphism.

To see that $\Psi$ is faithful, suppose that $\Psi\left(\xi^{i} \beta\right)$ is trivial for some $1 \leq i<r_{0}, \beta \in P\left(\pi^{\prime}\right)$. Then $\Psi\left(\xi^{i}\right)=\psi\left(\beta^{-1}\right)$. By considering the effect of the permutation on $1=(1, \ldots, 1)$, we find that $\beta$ must be 1 and hence the action of $\Psi\left(\xi^{i}\right)$ is trivial. It follows that $p$ has period $i<r_{0}$, contradicting the assumption that $r_{0}$ is the least period.

We summarize the above construction.

Lemma 2.6 Given a finite representation $p: \pi^{\prime} \rightarrow \Sigma$ of period $r$, there is a finite permutation representation $P: \pi_{1} X \rightarrow S_{N}$ such that $\left.P\right|_{\pi^{\prime}}$ is $r$-periodic and transitive. Moreover, $\left|P\left(\pi^{\prime}\right)\right|=N \geq\left|p\left(\pi^{\prime}\right)\right|$.

\subsection{Twisted Alexander polynomials induced by periodic representations}

Throughout this section, $P: \pi \rightarrow S_{N}$ is assumed to be a permutation representation induced by a finite representation $p: \pi^{\prime} \rightarrow \Sigma$ of period $r$, as in Lemma 2.6.

The representation $P$ induces an action of $\pi$ on the standard basis $\mathcal{B}=\left\{e_{1}, \ldots, e_{N}\right\}$ for $V=\mathbb{Z}^{N}$. We obtain a representation $\rho: \pi \rightarrow G L(V)$. Let $\epsilon$ be the abelianization homomorphism $\pi \rightarrow\langle t \mid\rangle$ mapping $x \mapsto t$. A twisted chain complex $C_{*}(X ; \Lambda \otimes V)$ is defined as in Section 2.1.

The free $\mathbb{Z}[\pi]$-complex $C_{*}(\tilde{X})$ has a basis $\{\tilde{z}\}$ consisting of a single lift of each cell $z$ in $X$. Then $\left\{1 \otimes e_{i} \otimes \widetilde{z}\right\}$ is a basis for the free $\Lambda$-complex $C_{*}(X ; \Lambda \otimes V)$ (cf page 640 of [5]).

We will use the following lemma from [10].

Lemma 2.7 Suppose that $A$ is a finitely generated $\mathbb{Z}\left[t^{ \pm 1}\right]$-module admitting a square presentation matrix and has 0 th characteristic polynomial $\Delta(t)=c_{0} \prod\left(t-\alpha_{j}\right)$. Let $s=t^{r}$, for some positive integer $r$. Then the 0 th characteristic polynomial of $A$, regarded as a $\mathbb{Z}\left[s^{ \pm 1}\right]$-module, is $\widetilde{\Delta}(s)=c_{0}^{r} \prod\left(s-\alpha_{j}^{r}\right)$.

The map $P: \pi \rightarrow S_{N}$ restricts to a representation of the fundamental group $\pi^{\prime}$ of the universal abelian cover $X_{\infty}$. Let $\widehat{X}_{\infty}$ denote the induced $N$-fold cover. The $\Lambda$ modules $H_{1}\left(\hat{X}_{\infty}\right)$ and $H_{1}(X ; \Lambda \otimes V)$ are isomorphic by two applications of Shapiro's Lemma (see for example [4]).

Algebraic 83 Geometric Topology, Volume 6 (2006) 
Proposition $2.8 H_{1}\left(\hat{X}_{\infty}\right)$ is a finitely generated $\mathbb{Z}\left[s^{ \pm 1}\right]$-module with a square presentation matrix, where $s=t^{r}$.

Proof Construct $X_{\infty}$ in the standard way, splitting $X$ along the interior of Seifert surface $S$ to obtain a relative cobordism $\left(V ; S^{\prime}, S^{\prime \prime}\right)$ bounding two copies $S^{\prime}, S^{\prime \prime}$ of $S$. Then $X_{\infty}$ is obtained by gluing countably many copies $\left(V_{j} ; S_{j}^{\prime}, S_{j}^{\prime \prime}\right)$ end-to-end, identifying $S_{j}^{\prime \prime}$ with $S_{j+1}^{\prime}$, for each $j \in \mathbb{Z}$.

For each $j$, let $W_{j}=V_{j r} \cup \cdots \cup V_{j r+r-1}$ be the submanifold of $X_{\infty}$ bounding $S_{j r}^{\prime}$ and $S_{j r+r-1}^{\prime \prime}$. Then $X_{\infty}$ is the union of the $W_{j}$ 's, glued end-to-end. After lifting powers of the meridian of $k$, thereby constructing basepaths from $S_{0}^{\prime}$ to each $S_{j r}^{\prime} \subset W_{j}$, we can then regard each $\pi_{1} W_{j}$ as a subgroup of $\pi_{1} X_{\infty} \cong \pi^{\prime}$.

Conjugation by $x$ in the knot group induces an automorphism of $\pi^{\prime}$, and the $r$ th power maps $\pi_{1} W_{j}$ isomorphically to $\pi_{1} W_{j+1}$. Since $p$ has period $r$, we have $p\left(x^{-r} u x^{r}\right)=p(u)$ for all $u \in \pi^{\prime}$. Hence $P$ has the same image on each $\pi_{1} W_{j}$. By performing equivariant ambient 0 -surgery in $W_{j}$ to the lifted surfaces $\hat{S}_{j}^{\prime}$ (that is, adding appropriate hollow 1-handles to the surface), we can assume that the image of $P\left(\pi_{1} S_{j}^{\prime}\right)$ acts transitively, and hence each preimage $\hat{S}_{j}^{\prime} \subset \hat{X}_{\infty}$ is connected.

The covering space $\hat{X}_{\infty}$ is the union of countably many copies $\hat{W}_{j}$ of the lift $\hat{W}_{0}$ glued end-to-end. The cobordism $\hat{W}_{0}$, which bounds two copies $\hat{S}^{\prime}, \hat{S}^{\prime \prime}$ of the surface $\widehat{S}$, can be constructed from $\hat{S}^{\prime} \times I$ by attaching $1-$ and 2-handles in equal numbers. Consequently, $H_{1} \widehat{W}_{0}$ is a finitely generated abelian group with a presentation of deficiency $d$ (number of generators minus number of relators) equal to the rank of $H_{1} \widehat{S}^{\prime}$.

The $r$ th powers of covering transformations of $\hat{X}_{\infty}$ induce a $\mathbb{Z}\left[s^{ \pm 1}\right]-$ module structure on $H_{1} \hat{X}_{\infty}$. The Mayer-Vietoris theorem implies that the generators of $H_{1} \widehat{W}_{0}$ serve as generators for the module. Moreover, the relations of $H_{1} \widehat{W}_{0}$ together with $d$ relations arising from the boundary identifications become an equal number of relators.

Corollary 2.9 If $\Delta_{\rho}(t)=1$, then $H_{1}\left(\hat{X}_{\infty}\right)$ is trivial.

Proof Let $s=t^{r}$, and regard $H_{1}\left(\hat{X}_{\infty}\right)$ as a $\mathbb{Z}\left[s^{ \pm 1}\right]$-module. Since the module has a square presentation matrix, its order ideal is principal, generated by $\widetilde{\Delta}_{\rho}(s)$. Lemma 2.7 implies that $\widetilde{\Delta}_{\rho}(s)=1$. Hence the order ideal coincides with the coefficient ring $\mathbb{Z}\left[s, s^{-1}\right]$. However, the order ideal is contained in the annihilator of the module (see [2] or Theorem 3.1 of [3]). Thus $H_{1}\left(\hat{X}_{\infty}\right)$ is trivial.

Since $p$ factors through $\pi_{1} M_{r}$, so does $\left.P\right|_{\pi^{\prime}}$. Let $\widehat{M}_{r}$ denote the corresponding $N$-fold cover. 
Lemma 2.10 $H_{1} \widehat{M}_{r}$ is a quotient of $H_{1} \widehat{X}_{\infty} /\left(t^{r}-1\right) H_{1} \widehat{X}_{\infty}$.

Proof Recall that $\pi_{1} M_{r} \cong \pi^{\prime} /\left[\pi^{\prime}, x^{r}\right]$. Thus $\pi_{1} \widehat{M}_{r} \cong \operatorname{ker}\left(\left.P\right|_{\pi^{\prime}}\right) /\left[\pi^{\prime}, x^{r}\right]$, and by the Hurewicz theorem,

$$
H_{1} \widehat{M}_{r} \cong \operatorname{ker}\left(\left.P\right|_{\pi^{\prime}}\right) / \operatorname{ker}\left(\left.P\right|_{\pi^{\prime}}\right)^{\prime} \cdot\left[\pi^{\prime}, x^{r}\right] .
$$

On the other hand, $\pi_{1} \hat{X}_{\infty}$ modulo the relations $x^{-r} g x^{r}=g$ for all $g \in \pi_{1} \hat{X}_{\infty}$ is isomorphic to $\operatorname{ker}\left(\left.P\right|_{\pi^{\prime}}\right) /\left[\operatorname{ker}\left(\left.P\right|_{\pi^{\prime}}\right), x^{r}\right]$. Using the Hurewicz theorem again,

$$
H_{1} \hat{X}_{\infty} /\left(t^{r}-1\right) H_{1} \hat{X}_{\infty} \cong \operatorname{ker}\left(\left.P\right|_{\pi^{\prime}}\right) / \operatorname{ker}\left(\left.P\right|_{\pi^{\prime}}\right)^{\prime} \cdot\left[\operatorname{ker}\left(\left.P\right|_{\pi^{\prime}}\right), x^{r}\right] .
$$

The conclusion follows immediately.

Example 2.11 The group $\pi$ of the trefoil has presentation $\left\langle x, a \mid a x^{2} a=x a x\right\rangle$, where $x$ represents a meridian, and $a$ is in the commutator subgroup $\pi^{\prime}$. The ReidemeisterSchreier method yields the presentation

$$
\pi^{\prime}=\left\langle a_{j} \mid a_{j} a_{j+2}=a_{j+1}\right\rangle,
$$

where $a_{j}=x^{-j} a x^{j}$. Consider the homomorphism $p: \pi^{\prime} \rightarrow \Sigma=\left\langle\alpha \mid \alpha^{3}\right\rangle \cong \mathbb{Z}_{3}$ sending $a_{2 j} \mapsto \alpha$ and $a_{2 j+1} \mapsto \alpha^{2}$. We extend $p$ to $P: \pi \rightarrow \widehat{\Sigma}=\left\langle\xi \mid \xi^{2}\right\rangle \ltimes \Sigma^{2}$, sending $x \mapsto \xi$. The image $P\left(\pi^{\prime}\right)$ consists of the three elements $(1,1),\left(\alpha, \alpha^{2}\right),\left(\alpha^{2}, \alpha\right)$; the image of $\pi$ is isomorphic to the dihedral group $D_{3}$, which we regard as a subgroup of $G L_{3}(\mathbb{Z})$. Hence we have a representation $\rho: \pi \rightarrow G L_{3}(\mathbb{Z})$. Let $\epsilon: \pi \rightarrow\langle t \mid\rangle$ be the abelianization homomorphism mapping $x \mapsto t$. The product of $\rho$ and $\epsilon$ determines a tensor representation $\rho \otimes \epsilon: \pi \rightarrow G L_{3}\left(\mathbb{Z}\left[t^{ \pm 1}\right]\right)$ defined by $(\rho \otimes \epsilon)(g)=\rho(g) \epsilon(g)$, for $g \in \pi$. We order our basis so that:

$$
(\rho \otimes \epsilon)(x)=\left(\begin{array}{ccc}
0 & t & 0 \\
t & 0 & 0 \\
0 & 0 & t
\end{array}\right), \quad(\rho \otimes \epsilon)(a)=\left(\begin{array}{lll}
0 & 1 & 0 \\
0 & 0 & 1 \\
1 & 0 & 0
\end{array}\right)
$$

We can assume that the CW structure on $X$ contains a single 0 -cell $p, 1-$ cells $x, a$ and a single 2-cell $r$.

The $\rho$-twisted cellular chain complex $C_{*}(X ; \Lambda \otimes V)$ has the form

$$
0 \rightarrow C_{2} \cong \Lambda^{3} \stackrel{\partial_{2}}{\longrightarrow} C_{1} \cong \Lambda^{6} \stackrel{\partial_{1}}{\longrightarrow} C_{0} \cong \Lambda^{3} \rightarrow 0 .
$$

If we treat elements of $\Lambda^{3}$ and $\Lambda^{6}$ as row vectors, then the map $\partial_{2}$ is described by a $3 \times 6$ matrix obtained in the usual way from the $1 \times 2$ matrix of Fox free derivatives:

$$
\left(\begin{array}{ll}
\frac{\partial r}{\partial x} & \frac{\partial r}{\partial a}
\end{array}\right)=\left(a+a x-1-x a \quad 1+a x^{2}-x\right)
$$


replacing $x, a$ respectively with their images under $\rho \otimes \epsilon$. The result is:

$$
\partial_{2}=\left(\begin{array}{cccccc}
t-1 & 1 & -t & 1 & t^{2}-t & 0 \\
0 & -t-1 & t+1 & -t & 1 & t^{2} \\
1-t & t & -1 & t^{2} & 0 & 1-t
\end{array}\right)
$$

The map $\partial_{1}$ is determined by $(\rho \otimes \epsilon)(x)-I$ and $(\rho \otimes \epsilon)(a)-I$ :

$$
\partial_{1}=\left(\begin{array}{ccc}
-1 & t & 0 \\
t & -1 & 0 \\
0 & 0 & t-1 \\
-1 & 1 & 0 \\
0 & -1 & 1 \\
1 & 0 & -1
\end{array}\right)
$$

Dropping the first three columns of the matrix for $\partial_{2}$ produces a $3 \times 3$ matrix:

$$
A=\left(\begin{array}{ccc}
1 & t^{2}-t & 0 \\
-t & 1 & t^{2} \\
t^{2} & 0 & 1-t
\end{array}\right)
$$

Similarly, eliminating the last three rows of $\partial_{1}$ gives:

$$
B=\left(\begin{array}{ccc}
-1 & t & 0 \\
t & -1 & 0 \\
0 & 0 & t-1
\end{array}\right)
$$

Theorem 4.1 of [5] implies that $\Delta_{\rho}(t) / \Delta_{0}(t)=\operatorname{Det} A /$ Det $B$, where $\Delta_{0}(t)$ is the 0 th characteristic polynomial of $H_{0} \hat{X}_{\infty}$. Since $\hat{X}_{\infty}$ is connected, $\Delta_{0}(t)=t-1$. Hence $\Delta_{\rho}(t)=\left(t^{2}-t+1\right)\left(t^{2}-1\right)$.

In this example, the cyclic resultant $\operatorname{Res}\left(\Delta_{\rho}(t), t^{2}-1\right)$ vanishes, indicating that $H_{1} \hat{X}_{2}$ is infinite. A direct calculation reveals that in fact $H_{1} \hat{X}_{2} \cong \mathbb{Z} \oplus \mathbb{Z}$.

Remark 2.12 In the above example we see that the Alexander polynomial of the trefoil knot divides the twisted Alexander polynomial. Generally, the Alexander polynomial divides any twisted Alexander polynomial arising from a finite permutation representation of the knot group. A standard argument using the transfer homomorphism and the fact that $H_{1} X_{\infty}$ has no $\mathbb{Z}$-torsion shows that $H_{1} X_{\infty}$ embeds as a submodule in $H_{1} \hat{X}_{\infty}$. Hence $\Delta(t)$, which is the 0 th characteristic polynomial of $H_{1} X_{\infty}$, divides $\Delta_{\rho}(t)$, the 0 th characteristic polynomial of $H_{1} \hat{X}_{\infty}$. 


\section{Proof of the Theorem}

Alexander polynomials are a special case of twisted Alexander polynomials corresponding to the trivial representation. Hence it suffices to consider an arbitrary nontrivial knot $k$ with unit Alexander polynomial $\Delta(t)$.

A complete list of those finite groups that can act freely on a homology 3-sphere is given in [8]. The only nontrivial such group that is perfect (that is, has trivial abelianization) is the binary icosahedral group $A_{5}^{*}$, with order 120 .

Since $\Delta(t)$ annihilates $H_{1} X_{\infty}$, the condition that $\Delta(t)=1$ implies that $H_{1} X_{\infty}$ is trivial or equivalently, that $\pi^{\prime}$ is perfect. Hence each branched cover $M_{r}$ has perfect fundamental group and so is a homology sphere. Theorem 3.7 of [6] implies that for some integer $r>2$, the group $\pi_{1} M_{r}$ is "large" in the sense that it contains a finite-index subgroup with a free nonabelian quotient.

Any large group has normal subgroups of arbitrarily large finite index. Hence $\pi_{1} M_{r}$ contains a normal subgroup $Q$ of index $N_{0}$ exceeding 120. Composing the canonical projection $\pi^{\prime} \rightarrow \pi_{1} M_{r}$ with the quotient map $\pi_{1} M_{r} \rightarrow \pi_{1} M_{r} / Q=\Sigma$, we obtain a surjective homomorphism $p: \pi^{\prime} \rightarrow \Sigma$ of least period $r_{0}$ dividing $r$. By Proposition 2.3, we have $r_{0}>1$. Let $P$ be the extension to $\pi$, as in Lemma 2.6. By that lemma, the order $N$ of $P\left(\pi^{\prime}\right)$ is no less than $N_{0}=\left|p\left(\pi^{\prime}\right)\right|$.

As in section 2, realize $P(\pi)$ as a group of permutation matrices in $G L_{N}(\mathbb{Z})$ acting transitively on the standard basis of $\mathbb{Z}^{N}$. Let $\hat{M}_{r_{0}}$ be the cover of $M_{r_{0}}$ induced by the representation $P: \pi \rightarrow S_{N}$. The group of covering transformations acts freely on $\widehat{M}_{r_{0}}$ and transitively on any point-preimage of the projection $\widehat{M}_{r_{0}} \rightarrow M_{r_{0}}$. Its cardinality is equal $N$ and so cannot be the binary icosahedral group. Hence $\hat{M}_{r_{0}}$ has nontrivial homology.

Lemma 2.10 and Corollary 2.9 imply that $\Delta_{\rho}(t) \neq 1$.

\section{References}

[1] J C Cha, Fibred knots and twisted Alexander invariants, Trans. Amer. Math. Soc. 355 (2003) 4187-4200 MR1990582

[2] R H Crowell, The annihilator of a knot module, Proc. Amer. Math. Soc. 15 (1964) 696-700 MR0167976

[3] J Hillman, Algebraic invariants of links, Series on Knots and Everything 32, World Scientific Publishing Co., River Edge, NJ (2002) MR1932169 
[4] J A Hillman, C Livingston, S Naik, Twisted Alexander polynomials of periodic knots, Algebr. Geom. Topol. 6 (2006) 145-169 MR2199457

[5] P Kirk, C Livingston, Twisted Alexander invariants, Reidemeister torsion, and CassonGordon invariants, Topology 38 (1999) 635-661 MR1670420

[6] M Lackenby, Some 3-manifolds and 3-orbifolds with large fundamental group arXiv: math.GT/0507283

[7] XS Lin, Representations of knot groups and twisted Alexander polynomials, Acta Math. Sin. (Engl. Ser.) 17 (2001) 361-380 MR1852950

[8] J Milnor, Groups which act on $S^{n}$ without fixed points, Amer. J. Math. 79 (1957) 623-630 MR0090056

[9] D S Silver, S G Williams, Crowell's derived group and twisted polynomials, J. Knot Theory Ramifications (in press) arXiv:math.GT/0506339

[10] D S Silver, S G Williams, Lifting representations of $\mathbb{Z}$-groups, Israel J. Math. 152 (2006) 313-331 MR2214466

[11] M Wada, Twisted Alexander polynomial for finitely presentable groups, Topology 33 (1994) 241-256 MR1273784

Department of Mathematics and Statistics, University of South Alabama

Mobile, AL 36688-0002, USA

silver@jaguar1.usouthal.edu, swilliam@jaguar1.usouthal.edu

Received: 5 June 2006 Revised: 20 August 2006 